\title{
Analysis on Equity of China Medical Resources Allocation
}

\section{------the Case of Shanghai}

\author{
Di Sun ${ }^{1}$ \\ ${ }^{1}$ School of Management, Shanghai University of Engineering Science, China \\ Correspondence: Di Sun, School of Management, Shanghai University of Engineering Science, Shanghai 201620, \\ China. Tel: 86-188-1823-3673. E-mail:sdclevergirl@163.com
}

Received: September 20, 2013

Accepted: October 8, 2013

Online Published: October 9, 2013

doi:10.5430/jbar.v2n2p61

URL: http://dx.doi.org/10.5430/jbar.v2n2p61

The number of the innovative program: A-0903-13-01068.

\begin{abstract}
Shanghai launched a wilding heath system reform from 2010 due to the unreasonable medical allocation between city and suburb that restricts the equalization of medical service. Under this background, the research finds that the gap between city and suburb is considerable big. So the paper uses the data of resident population and the means of Lorenz curve and Gini coefficient to analyze the equity of medical resources allocation among seventeen counties in Shanghai. As a result, from the point of population allocation, it is relative equity, but it is not visible equity between city and suburb. Therefore, the government should increase investment to the area of lack of medical resources which lies in suburb. And according to the market demand to allocate the medical resources, so gradually improve the health level of the residents, and reduce the pressure of the difficulty and high prices about seeing a doctor.
\end{abstract}

Keywords: Medical resource allocation, Resident population, Equity, Lorenz curve

\section{Introduction}

Shanghai is one of China's fastest growing cities, which has advanced medical equipment and medical resources. However, the allocation of medical resources is not reasonable in Shanghai, which hindered the realization of the right to equal access to health services. Therefore Shanghai carried out the deepening medical and health system reform in 2011.Unreasonable allocation of medical resources is mainly reflected in city which has abundant medical resources, advanced medical equipment and excellent medical personnel, however the distribution of medical resources in the suburbs is less. At the same time, the city not only absorbs the local residents, but also the surrounding population, increasing the burden of the city medical resources. Based on the 2012 Shanghai Statistical Yearbook data and related information, using the method of Lorenz curve and the Gene coefficient, to study the fairness of allocation of medical resources, the present allocation of health resources in Shanghai and the existing problems in order to get new discoveries and conclusions.

\section{The allocation of medical resources in Shanghai}

According to the Shanghai Statistical Yearbook 2012, Shanghai constantly enrich the optimization of medical resources, since the number of beds 58400 in 1981 to 107,100 in 2011, an increase of more than doubled. Health technicians from 9.57 million in 1981, increased to 13.91 million in 2011, an increase of $45 \%$; the number of medical institutions from 6337 in 1981 dropped to 3358 in 2011, reduced by nearly half. Shanghai's GDP rose from 32.476 billion to 1919.569 billion in 2011, an increase of 59 times, as the figure 1 shows. 


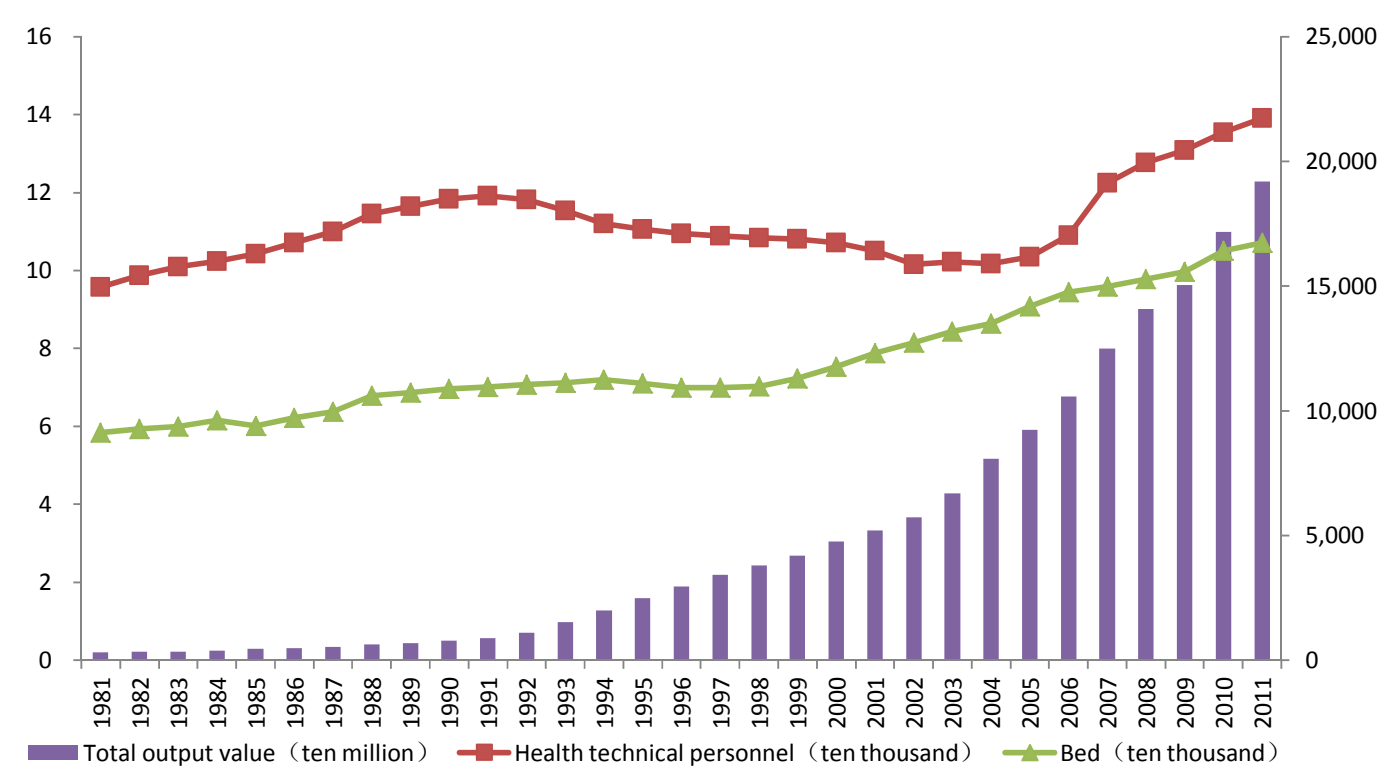

Figure 1. 1981-2011 the number of health institutions, the beds, the health workers and GDP

Data source: Shanghai Statistics Bureau

From the figure 1, we see that health professionals, health beds and Shanghai's GDP show a rising trend overall, but Shanghai's GDP growth rate is far greater than the growth rate of health care resources. Figure 2 shows, Shanghai government on health expenditure has little difference in volume from 1991 to 2005, however, the investments in health cost have increased significantly from 2006 to 2011. Under this background, the selection of medical resources are mainly four factors to analysis the medical resources allocation, namely medical institutions, beds, health and technical personnel, the number of doctors.

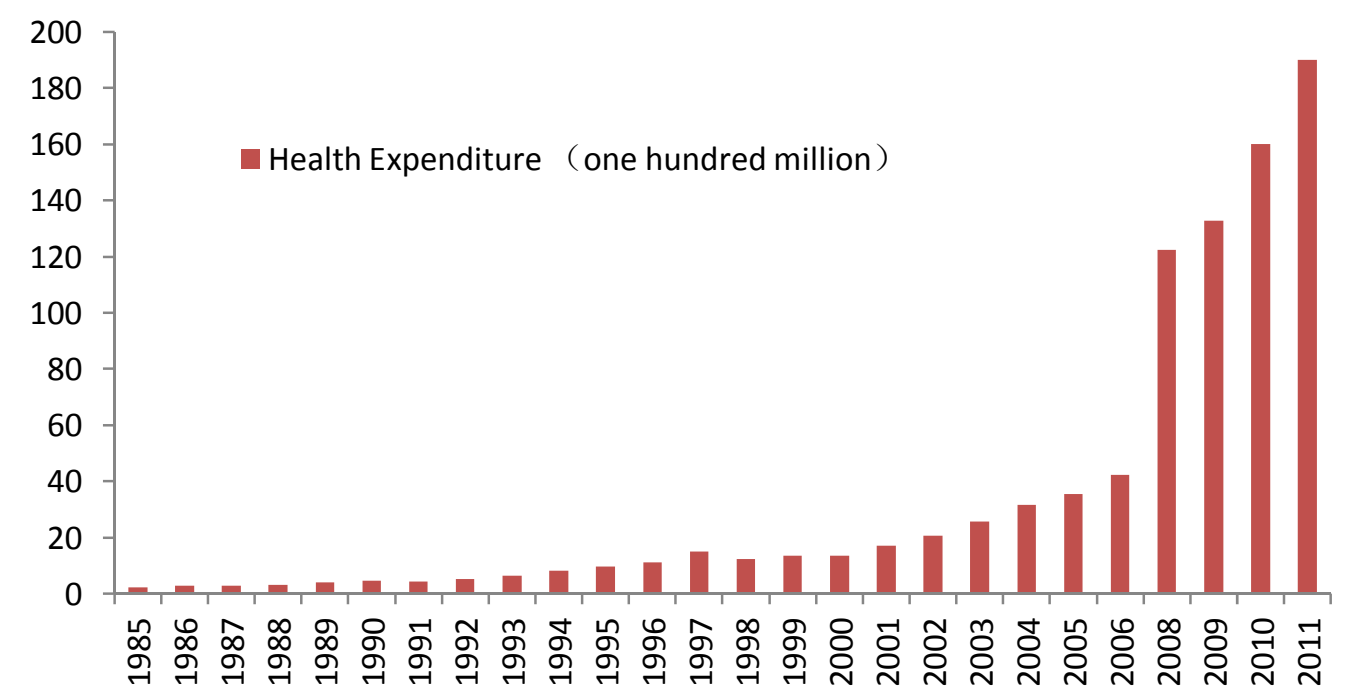

Figure 2. 1985-2011 Shanghai financial situation of the health investment

Data source: Shanghai Statistics Bureau

\section{The problem of allocation of medical resources}

\subsection{Human Resources for Health in Shanghai inclined to city seriously}

From Table 1, it can be found center city $3.94 \%$ of the population, health professionals and the percentage of doctors who were $17.09 \%, 16.29 \%$, it has a relative surplus of medical resources; suburban areas domain the percentage of population is $32.27 \%$, the suburb is $15.78 \%$, respectively the percentage of health workers are $18.13 \%, 11.24 \%$, the percentage of doctors who are $19.28 \%, 11.52 \%$, and doctors in health personnel configuration are low. 
Table 1. The distribution of medical resources during the five area in Shanghai

\begin{tabular}{llllllll}
\hline & $\begin{array}{l}\text { Population } \\
(\%)\end{array}$ & Area (\%) & Institution (\%) & $\begin{array}{l}\text { Bed } \\
(\%)\end{array}$ & $\begin{array}{l}\text { Health } \\
(\%)\end{array}$ & workers & $\begin{array}{l}\text { Doctor } \\
(\%)\end{array}$ \\
\hline Pudong & 22.05 & 19.09 & 17.69 & 14.35 & 14.41 & 15.14 \\
Center city & 3.94 & 0.44 & 10.63 & 14.45 & 17.09 & 16.29 \\
Centers around the city & 25.97 & 4.12 & 32.37 & 39.87 & 39.13 & 37.77 \\
Suburban area & 32.27 & 26.99 & 26.89 & 18.03 & 18.13 & 19.28 \\
Suburbs & 15.78 & 49.35 & 12.42 & 13.3 & 11.24 & 11.52 \\
\hline
\end{tabular}

Data source: according to Shanghai Statistics Bureau

\subsection{The allocation standard of medical resources is not reasonable}

Medical institutions in Shanghai are not in accordance with the population distribution area, but according to the administrative relations and department layout. Precisely, such a configuration standard medical resources results it, which mainly gathered in the economically developed regions, and for economically disadvantaged areas are relatively scarce medical resources. As the increasing number of fluid population in Shanghai, the demand for health care resources more than ever. According to the 2012 Statistical Yearbook shows that in 2011, the population in rural and suburban reached 1,645.45 million, the city population was 702.01 million, rural and suburban population was twice than the city population. This leads to a large number of population in rural and suburb lack of medical resources, and the city population are relatively small, but over supply of medical resources.

\section{Analysis on equity of medical resources allocation among city and suburb in Shanghai}

To analyze the fairness of health resource allocation, Wang Ling (2012) uses longitudinal method to analyze the data from 2004 to 2008. Dai Yingzi(2010) from the perspective of non-equilibrium analysis to analyze medical resources allocation. There are many similar examples to analyze the fairness of health resource allocation, such as Guo Zhenyou (2012) and Zhang Lu-lu(2000) also use the Lorenz curve and the Gini coefficient method to analyze health resource allocation about the fairness. Li Xiaorong (2012) put forward the idea that bases on the network organization theory to build Yunnan health care system, which can optimize the allocation of medical resources. Wang Shu(2010) according to Herman Harken synergy theory, discussed the regional medical resource allocation movement and dynamic mechanism, for building regional cooperative healthcare system and optimizing the allocation of medical resources provide a theoretical reference.

\subsection{Research Methods}

Lorenz curve was proposed by American statistician, that in a general (country, region), from the poorest population to calculate the percentage of revenue to the percentage of the richest population" corresponding to each population percentage points of the curve. It bends the greater, the degree distribution more unequal, and vice versa. Gini Coefficient proposed by the Italian economists guineas, quantitative determination of the degree of difference distribution, with a value between 0 and 1. In accordance with the provisions of relevant United Nations organizations: less than 0.2 indicates the absolute average; 0.2 to 0.3 indicates relatively average; 0.3 - 0.4 indicates a relatively reasonable; $0.4-0.5$ indicates a big gap between above; 0.6 indicates the disparity, economists typically take 0.4 as the allocation gap "warning."

\subsection{Research content}

Depending on geographic location and economic development, we divide the 17 administrative regions into five regions: Pudong; suburbs, including Jinshan, Qingpu, Fengxian and Chongming County; suburban areas, including Baoshan, Minhang, Jiading and Songjiang; centers around the city, including the Xuhui, Changning, Putuo, Zhabei, Hongkou and Yangpu; central city, including Huangpu and Jing'an.

Let's analyze the distribution of medical resources in Shanghai. In the calculation of per capita health care resources, which are basically based on household population, without taking the floating population into account. The paper makes up this point. Through the survey, we find that the number of most resident population is larger than the household population (see Figure 3). 


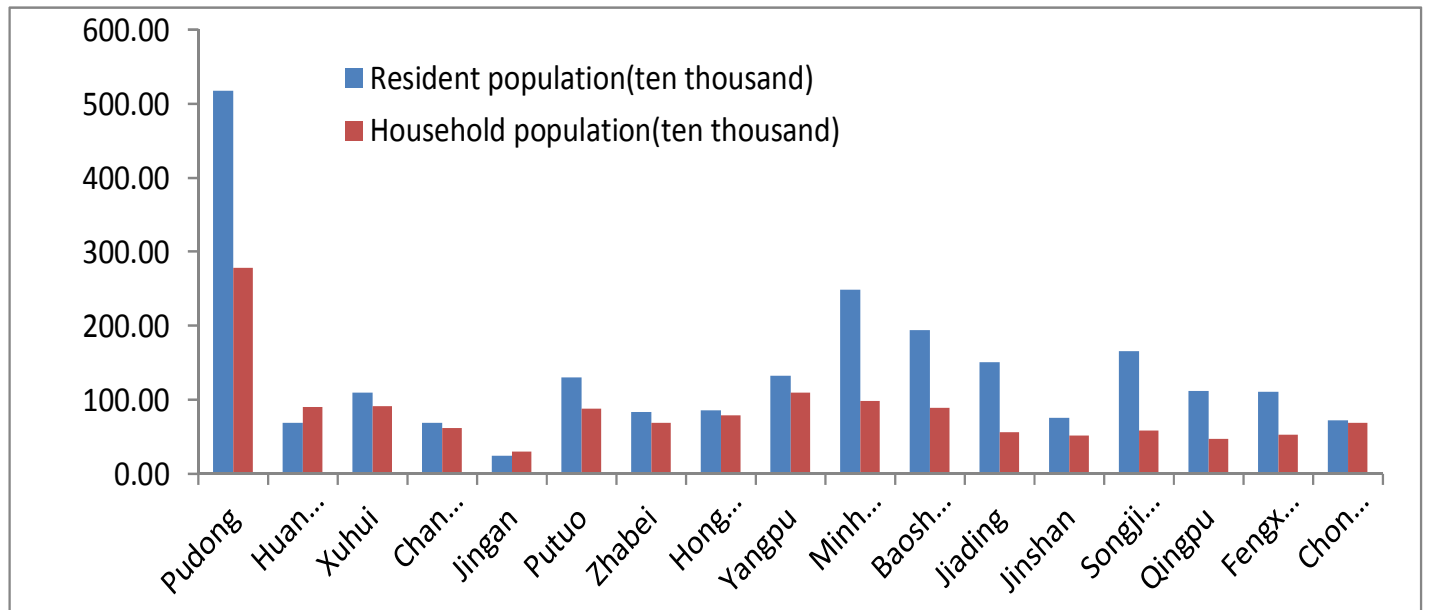

Figure 3. At the end of 2011, the resident population and the household population in Shanghai

Data source: Shanghai Statistics Bureau,http://www.stats-sh.gov.cn/tjnj/nj12.htm?d1=2012tjnj/C2101.htm

This paper mainly uses four measuring elements to study the equity in health care resources, that is, the number of medical institutions, professionals, beds and doctors. According to the population cumulative percentage and the cumulative percentage of medical resources, draw Lorenz curve of population distribution in Shanghai in 2011(see Figure 4); according to the area cumulative percentage and cumulative percentage of medical resources, draw Lorenz curve of area distribution in Shanghai in 2011(see Figure 5). According to Lorenz curve, use the method of geometric estimation to draw the Gene coefficient curve.

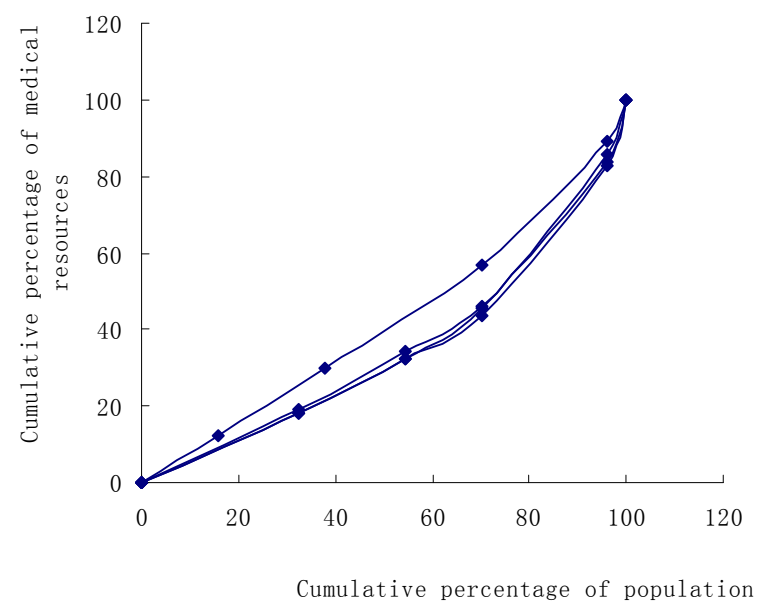

Figure 4. Shanghai medical resources configured by population Lorenz curve

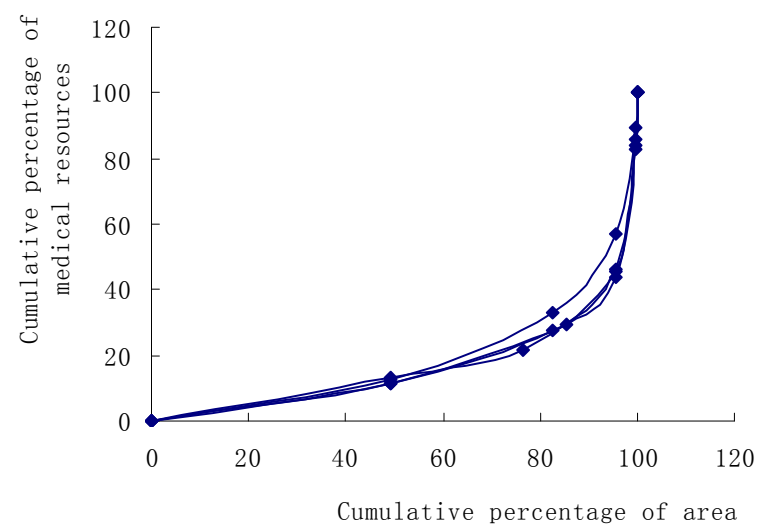

Figure 5. Shanghai medical resources configured by areas Lorenz curve 
According to figure 4, the medical resources base on the population distribution is relatively fair, however, the area of medical resources allocation is not fair. From table 1, the health technical personal is higher than any other medical resources which indicates its distribute needs to improve. Compared with resident population, the allocation of medical resources base on the household population is better (see Table 2).

Table 2. The Gini coefficient comparison of resident and household population in Shanghai, 2011

\begin{tabular}{lll}
\hline Medical resources categories & Resident population & Household population \\
\hline Bed & 0.2937 & 0.1328 \\
Doctor & 0.2836 & 0.1408 \\
Medical institution & 0.1509 & 0.0882 \\
Health technical personnel & 0.3084 & 0.1616 \\
\hline
\end{tabular}

Data source: according to Shanghai Statistics Bureau

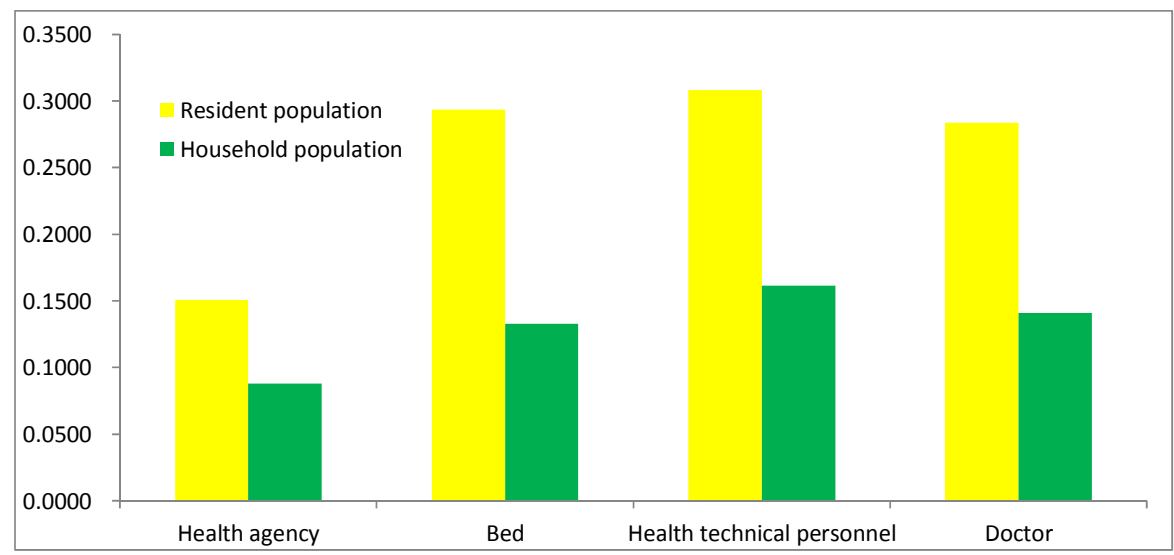

\section{The suggestion about optimizing the allocation of medical resources}

This paper argues that the allocation of medical resources should not simply be based on the division of administrative regions configuration, but according to market supply and demand, and guide medical resources between regions to achieve effective configuration. Meanwhile, given the government macro-control and the development of relevant policy documents, the government should guide the allocation of medical resources to achieve rational allocation between the suburbs and increases the investment to the suburbs, step by step, to achieve universal access to health services equalization.

\section{References}

Dai Yingzi. (2010). On behalf of heroic urban medical resource allocation: Non-Equilibrium and Correction. Urban Development, v.09:108-112.

Guozhen You \& Dan Wuxiang. (2012). 2000 to 2008 the fair allocation of health resources research. Chinese Health Statistics, v. 2902:224-225.

Li Xiaorong, Gu Youjin \& Liang Wuchao. (2012). Western frontier minority areas allocation of medical resources status and problems -A case study. Academic Exploration, v.06:113-115.

Luo Juan, Wang Hong \& Cui Kaichang. (2009). Shanghai Medical Resource Allocation Analysis. Chinese Health Statistics, v.05:466 -469.

Qi Mingzhu \& Tong Yufen. (2010). Allocation of medical resources between districts and counties of Beijing Population Study of Fairness. Beijing Social Sciences, v.05:27-33.

Wang Ling \& Zhang Xiang. (2010). $2004 \sim 2008$ Shanghai Health Resources Equity Research. Chinese Health Service Management, v.27; No.26002:85-87.

Wang Shu, Wang Heng \& Wang Yunguang. (2010). Research on Synergetic Dynamical Mechanism for Regional Cooperative Healthcare System for Medical Resources Optimization Deployment. Science and Technology Management Research, v.13:270-275.

Zhang Lu-lu, Hu Shan-lian \& Wei Ying. (2010). Area efficiency of resource allocation within the hospital medical research. Chinese Hospital Management, v.05:14 -16. 